IN THIS ISSUE

The P\&P Hairline Laser: A Novel HandsFree Laser-Assisted Hairline Device for Hair Restoration Surgery

Treating Hair Loss Caused by Synthetic Hair Implant

One-Month Trial of HOCl: An Observational Pilot Study

Anesthetic Infiltration with Microcannulas

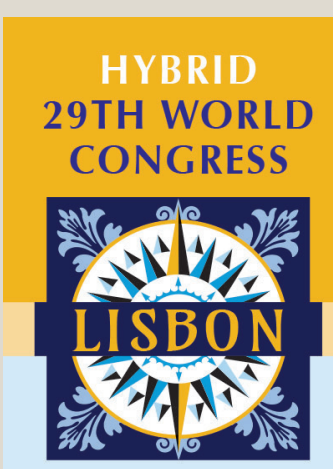

October 21-23, 2021

29thannual.org

Don't Miss

The Big One!

INTERNATIONAL SOCIETY OF HAIR RESTORATION SURGERY

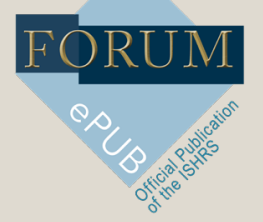

\section{A Study Comparing Survival of Hair Follicles Stored Cold and at Room Temperature}

\author{
Michael L. Beehner, MD II Saratoga Springs, New York, USA I mlbeehner@saratogahair.com
}

\section{ABSTRACT}

Prior to being inserted in the recipient site, grafts are typically stored for hours ex vivo at a cold temperature $\left(4^{\circ} \mathrm{C}\right)$. However, previous research has suggested that room temperature storage may deliver similar results. Here, I describe a study on three patients to examine whether grafts (2-hair follicles each) have better survival after being stored in a cold $\left(4^{\circ} \mathrm{C}, 330\right.$ hair follicles) or a room-temperature $\left(21^{\circ} \mathrm{C}, 330\right.$ hair follicles) environment during surgery. I additionally examined whether hair follicles that received one of six different stressors (800 follicles) could be "rescued" by cold or room temperature storage. Results demonstrated that the follicles stored at room temperature survived significantly better $(90.9 \%)$ than those stored at a cold temperature $(80.3 \%)$. Neither temperature proved better for follicles that were stressed. In sum, chilling our graft storage solution may not be the best choice when we can achieve consistent superior survival with room-temperature storage.

Keywords: chilled, graft, hair transplant, hydrogen peroxide, Plasma-Lyte, solution storage, stress

This article is open access and may not be copied, distributed, or modified without written permission from the International Society of Hair Restoration Surgery.

\section{INTRODUCTION}

During the past 60 years, since Orentreich's introduction of hair transplantation as a cosmetic procedure for hair loss, the most common technique for storing hair grafts during surgery has been to place them in a chilled saline solution (or its near equivalent). ${ }^{2}$ The grafts are ex vivo from the time of removal from the donor site until they are inserted into the recipient sites. It has always been assumed that since large organs such as kidneys and hearts are kept cold until placed in the body, hair follicles would also survive better if kept chilled.

The temperature of graft storage has been investigated in recent years, but the combined results have not been conclusive. Raposio and colleagues dissected 240 1-hair follicles and placed half of them in room-temperature saline and the other half in cold normal saline $\left(1^{\circ} \mathrm{C}\right)$ for 5 hours to replicate the time out of body during a normal transplant procedure. ${ }^{3}$ They then cultured follicles in a supplemental medium to mimic an in vivo environment. After 10 days, they evaluated the growth of these follicles, sorting them into those that grew any length at all and those that did not grow. They showed that $87 \%$ of the follicles cultured at room temperature and $88 \%$ of the cold-stored ones showed some microscopic growth. Given nearly identical rates of growth, they concluded that there was no difference between the temperature storage effects. However, this study was conducted entirely out of a human body and extended only 10 days into hair growth.

One of the only other studies to specifically examine temperature storage was conducted in a similar way using growth/no growth after being cultured in a dish for a length of time. Kim and colleagues (who also examined dehydration of follicles) found that follicles stored at room temperature and those stored at $4^{\circ} \mathrm{C}$ showed no difference in growth when stored up to 6 hours (however, cold storage did increase growth for those stored up to 48 hours). They concluded that, as long as grafts were not mishandled and kept hydrated, cooling grafts during storage was not necessary and only helped survival if the operation time extended over 6 hours. ${ }^{4}$

In a similar study, I showed that chilled grafts seemed to tolerate "stress" (crushing trauma) better than grafts maintained at room temperature (followed up by a study showing that chilling tissue in Hypo- 
Thermosol ${ }^{\circledR}$ and ATP resulted in a higher yield of hair ${ }^{5}$ ). However, what appears to be lacking from the literature is a study on temperature storage conditions for "non-stressed" follicles conducted on actual patients to assess growth rate.

Here, I report the results from a 3-year study of three male patients where I compared the $\sim 1$-year survival of grafts stored cold (5 hours) compared to grafts stored at room temperature (5 hours). To assess the additional effects of "stress," I also added several treatment conditions to some of the temperature storage conditions.

\section{MATERIALS AND METHODS}

Three male subjects ages 41-64 (two with bald frontal areas, one with Norwood Class VI balding) were recruited to examine the effects of storing follicles (5 hours) in either cold Plasma-Lyte $\left(4^{\circ} \mathrm{C}\right)$ or room-temperature Plasma-Lyte $\left(21^{\circ} \mathrm{C}\right)$. The focus of this study was to compare whether grafts under normal conditions (no stress) survived better in cold or in room-temperature storage solution (660 hair follicles divided evenly between the two temperature conditions).

I additionally evaluated whether the temperature of the holding solution in surgery had any effect on survival of grafts that received a stress treatment (an additional 800 hair follicles; Table 1). The idea was to ascertain if temperature interacted with trauma conditions to improve survival (e.g., if a cold temperature might rescue a follicle treated with $3 \%$ hydrogen peroxide). The description of each treatment condition is as follows:

- None: These follicles did not receive a "stressor" treatment condition, only a temperature storage condition.

- Hydrogen peroxide (1\%): I dunked each follicle into $1 \%$ hydrogen peroxide for 80 seconds, followed by temperature storage conditions.

- Bulb crushed (hard): I crushed the bulb of the hair follicle using needle-nose pliers, followed by temperature storage conditions.

FIGURE 1. Needle-nose pliers with a soft rubber cover was used for "soft" crush treatments (bulb and bulge). Photo: Michael L. Beehner

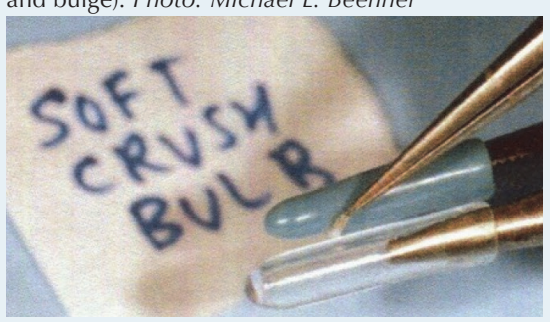

- Bulb crushed (soft): I crushed the bulb of the hair follicle using needle-nose pliers with a soft rubber cover around the tip (Figure 1), followed by temperature storage conditions.

TABLE 1. Number of Follicles Placed and Treatment Condition and Temperature Storage Conditions (stored cold $\left[4^{\circ} \mathrm{C}\right]$ or room temperature $\left[21^{\circ} \mathrm{C}\right]$ )

\begin{tabular}{|c|c|c|c|c|}
\hline Patient & Treatment condition & $\begin{array}{r}\text { Temperature storage } \\
\text { COLD }\end{array}$ & $\begin{array}{r}\text { Temperature storage } \\
\text { ROOMTEMPP }\end{array}$ & $\begin{array}{r}\text { Total } \\
\text { follicles }\end{array}$ \\
\hline \multirow[t]{7}{*}{1} & None & 50 & 50 & 100 \\
\hline & Hydrogen Peroxide $(1 \%)$ & 50 & 50 & 100 \\
\hline & Bulb crushed (hard) & 50 & 50 & 100 \\
\hline & Bulb crushed (soft) & 50 & 50 & 100 \\
\hline & Bulge crushed (soft) & 50 & 50 & 100 \\
\hline & Stored dry (gauze) & 50 & 50 & 100 \\
\hline & Hydrogen Peroxide $(3 \%)$ & 50 & 50 & 100 \\
\hline \multirow[t]{2}{*}{2} & None & 100 & 100 & 200 \\
\hline & Hydrogen Peroxide (3\%) & 100 & 100 & 200 \\
\hline 3 & None & 180 & 180 & 360 \\
\hline
\end{tabular}

- Bulge crushed (soft): I crushed the bulge of the hair follicle using needle-nose pliers with a soft rubber cover around the tip, followed by temperature storage conditions.

- Stored dry (gauze): I stored the hair follicle on dry gauze for 18 minutes, followed by temperature storage conditions.

- Hydrogen peroxide (3\%): I dunked each follicle into $3 \%$ hydrogen peroxide for 80 seconds, followed by temperature storage conditions.

Patient 1 received all stressor treatment conditions and both temperature storage conditions (cold, room temperature); patient 2 received one stressor treatment condition (3\% hydrogen peroxide) and both temperature storage conditions; and patient 3 received no stressor treatment conditions and both temperature storage conditions (Table 1). All patients provided written informed consent for the procedures indicated here. The 3-year study was conducted sequentially on one patient at a time. The original research focus was to examine a combination of stress conditions and temperature storage, but when it became clear that the stress conditions did not interact with temperature, I shifted the focus almost entirely to temperature storage (patients 2 and 3).

For the placement of the grafts, the study boxes were 1.1 $\times 1.1 \mathrm{~cm}$ for patients 1 and 2 , and $1.2 \times 1.2 \mathrm{~cm}$ for patient 3 . Each study box was marked with a tiny tattoo dot at each of the four corners. I left a $1.5 \mathrm{~mm}$-wide "moat" of bald scalp around each study box to facilitate easier and faster counting of follicles (Figure 2). I used $1 \mathrm{~mm}$-wide lateral slit blades to make sagittal recipient sites. All follicular unit (FU) grafts in the study were 2-hair FUs. The study grafts were out of the body for approximately 4-5 hours FIGURE 2. For all patients, a $1.5 \mathrm{~mm}$-wide "moat" of bald scalp was left around each study box to facilitate the for each procedure. All follicles $(1,460$ in total) were left in their recipient sites to be counted for the presence of a growing hair approximately 1 year later.

The hair counts

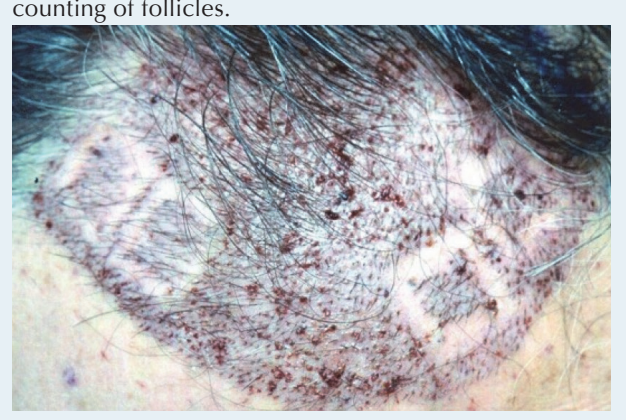

on the three patients were performed respectively at 8-, 12-, and 13-months post-surgery. The density of planting these 2-hair FUs were as follows:

Patient 1 - $25 \mathrm{FU} / 1.1 \times 1.1 \mathrm{~cm}$

Patient $2-25 \mathrm{FU} / 1.1 \times 1.1 \mathrm{~cm}$

Patient $3-45 \mathrm{FU} / 1.2 \times 1.2 \mathrm{~cm}$

When conducting final hair counts, fine vellous hairs were not included in final counts but the slightly "wispy" ones were (these accounted for $\sim 5-6 \%$ of the final counts). These percentages were nearly equal for all study boxes counted. All remaining hairs in all three patients were full terminal hairs.

Although each FU contained two hairs, I treated each hair follicle separately for assigning "survival" or "no survival" 
at the time of the final count. I calculated percentages for survival based on the number of hair follicles that survived/ number of hair follicles that were transplanted (Table 2). To ascertain statistical significance, I constructed a logistic regression model (a general linear model in the binomial family) with these data. The outcome variable was Survival (whether each hair follicle survived or not when counted nearly 1 year later). The predictor variables were Patient $(1,2,3)$, Treatment Condition (hydrogen peroxide [1\%], bulb crushed [hard], bulb crushed [soft], bulge crushed [soft], stored dry [gauze], and hydrogen peroxide [3\%]), and Temperature Storage (cold, room temperature). Because in this first model all treatment conditions except hydrogen peroxide (1\%) showed lower survival (regardless of temperature condition), I conducted a second model where I collapsed all treatment conditions except hydrogen peroxide $(1 \%)$ into one treatment group called "Stress" (i.e., the treatment groups in this model were "None," "Hydrogen Peroxide [1\%]," and "Stress"). For this second model, an interaction term was added between Temperature Storage and Treatment Condition to see if temperature storage could rescue the negative effects of a stressor. Results for the second model only are reported here. All statistics were run in $\mathrm{R}$ (4.0.5).

\begin{tabular}{|c|c|c|c|c|c|c|c|}
\hline Patient & Treatment condition & $\begin{array}{r}\text { Total } \\
\text { COLD TEMPP }\end{array}$ & $\begin{array}{r}\text { Survival } \\
\text { COLD TEMP }\end{array}$ & $\begin{array}{l}\text { Percent survival } \\
\text { COLD TEMP }\end{array}$ & $\begin{array}{r}\text { Total } \\
\text { ROOM TEMP }\end{array}$ & $\begin{array}{r}\text { Survival } \\
\text { ROOM TEMP }\end{array}$ & $\begin{array}{r}\text { Percent survival } \\
\text { ROOM TEMP }\end{array}$ \\
\hline \multirow[t]{7}{*}{1} & None & 50 & 39 & 0.78 & 50 & 46 & 0.92 \\
\hline & Hydrogen Peroxide $(1 \%)$ & 50 & 43 & 0.86 & 50 & 51 & 1.02 \\
\hline & Bulb crushed (hard) & 50 & 9 & 0.18 & 50 & 27 & 0.54 \\
\hline & Bulb crushed (soft) & 50 & 34 & 0.68 & 50 & 37 & 0.74 \\
\hline & Bulge crushed (soft) & 50 & 24 & 0.48 & 50 & 39 & 0.78 \\
\hline & Stored dry (gauze) & 50 & 40 & 0.80 & 50 & 37 & 0.74 \\
\hline & Hydrogen Peroxide $(3 \%)$ & 50 & 30 & 0.60 & 50 & 29 & 0.58 \\
\hline \multirow[t]{2}{*}{2} & None & 100 & 83 & 0.83 & 100 & 92 & 0.92 \\
\hline & Hydrogen Peroxide $(3 \%)$ & 100 & 40 & 0.40 & 100 & 55 & 0.55 \\
\hline 3 & None & 180 & 146 & 0.81 & 180 & 166 & 0.92 \\
\hline
\end{tabular}

\section{RESULTS}

After controlling for patient identity, there was a significantly higher chance of hair follicle survival $($ Estimate $=0.998, \mathrm{SE}=0.249, \mathrm{Z}$ value $=4.017, \mathrm{p}<0.001)$ approximately one year later for follicles stored in roomtemperature Plasma-Lyte $\left(21^{\circ} \mathrm{C}\right)$ compared to those stored in cold Plasma-Lyte $\left(4^{\circ} \mathrm{C}\right.$, Figure 3$)$. The follicles immersed in a room-temperature solution survived at a $90.9 \%$ rate, whereas those immersed in chilled solution survived at an $80.3 \%$ rate. This significant result held true for hair follicles with no treatment condition ("None") and those that were dunked in $1 \%$ hydrogen peroxide. However, the improved survival for room-temperature storage disappeared entirely under all "Stress" treatment conditions, as the interaction term failed to reach significance in our second model (Estimate $=-0.399, \mathrm{SE}=0.289, \mathrm{Z}$ value $=-1.379, \mathrm{p}=0.168)$. When lumped together, the "Stress" treatment condition caused a significant reduction in survival for all hair follicles (Estimate $=-1.558, \mathrm{SE}=0.229, \mathrm{Z}$ value $=-6.779, \mathrm{p}<0.001)$, and this decrease was unable to be "rescued" by cold or room-temperature storage (Figure 3).
FIGURE 3. The chart shows the proportion of hair follicles that survived (all three patients combined) for follicles stored ex vivo at different temperatures. The treatment conditions include: "None" = no treatment other than the temperature storage conditions; " $\mathrm{H} 2 \mathrm{O} 2-1 \%$ " = dunked in $1 \%$ hydrogen peroxide, then subjected to the temperature storage conditions; and "Stress" = all other treatment conditions combined, then subjected to the temperature storage conditions. Note that the proportion of surviving hair follicles is higher for those stored at room temperature in all but the "Stress" conditions.

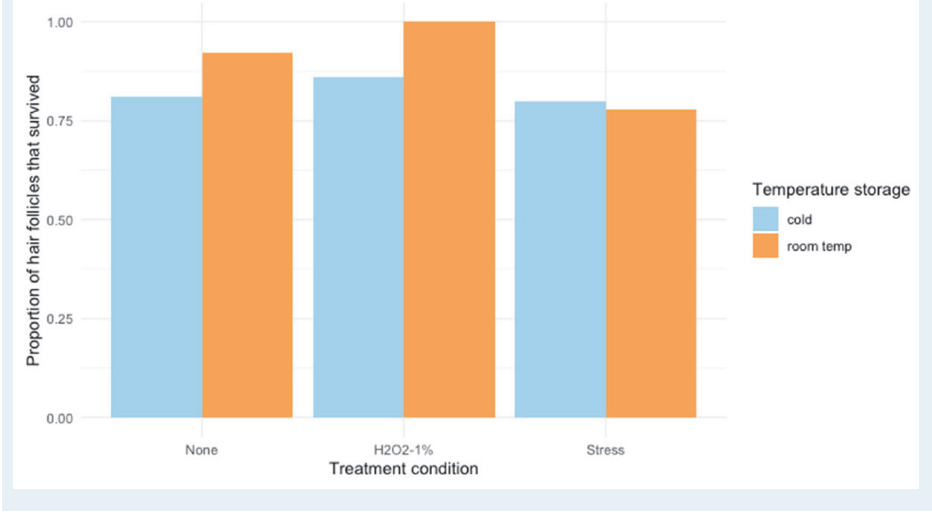

\section{DISCUSSION}

The results of this study strongly suggest that chilling graft storage solution may not be the best choice when we can achieve consistent superior survival when using a room-temperature storage solution. One explanation for this difference in survival may be that the sudden change in temperature from the relative warmth of the human scalp to the coolness of the petri dish is too jarring to the metabolic machinery of the follicle and scalp. Parsley appears to have picked up on this himself, as he reports that he first moves his hair follicles to a room-temperature dish prior to transferring them to an ice-based system to achieve a more gradual decline in temperature for the follicles (Parsley as cited in Cole and Reed). ${ }^{6}$ The room-temperature advantage disappeared when grafts were traumatized chemically or mechanically.

Finally, although this study was not intended to examine the effects of storage in a $1 \%$ hydrogen peroxide solution, it is interesting to note that the results hint at the possibility of there being some benefits to this solution that appear here, especially when combined with room-temperature Plasma-Lyte temperature storage.

\section{References}

1. Orentreich N. Autografts in alopecias and other selected dermatological conditions. Ann NY Acad Sci. 1959; 83(3):463-479.

2. Unger WP. Hair Transplantation, 3rd Ed. New York: Marcel Dekker, 1995.

3. Raposio $\mathrm{E}$, et al. Effects of cooling micrografts in hair transplantation surgery. Derm Surg. 1999; 25(9):705-707.

4. Kim JC, et al. The effects of dehydration, preservation temperature and time on the hair grafts. Ann Dermatol. 2002; 14(3):149-152.

5. Beehner M. Two research studies on follicular unit growth. Orlando Live Surgery Workshop, Orlando, Florida, USA; April 2002.

6. Cole JP, Reed WM. The optimal holding solution and temperature for hair follicle grafts. Hair Transp Forum Int'l. 2012; 22(1):17-21. 